\title{
A New Dilation Model for FRP Fully/partially Confined Concrete Column under Axial Loading
}

\author{
Javad Shayanfar ${ }^{1}$, Mohammadali Rezazadeh ${ }^{1}$, Joaquim Barros ${ }^{1}$, and Honeyeh \\ Ramezansefat $^{1}$ \\ ${ }^{1}$ ISISE, Dept. of Civil Engineering, Univ. of Minho, 4800-058 Guimarães, Portugal \\ $\operatorname{arch} 3 \mathrm{~d}$.ir@gmail.com
}

\begin{abstract}
Experimental research has confirmed that the usage of fiber reinforced polymer (FRP) composite materials can be a reliable solution to substantially improve axial and dilation behavior of confined concrete columns. In this regard, FRP partial confinement system is a good compromise from the cost competitiveness point of the view, while the application of discrete FRP strips provides less confinement efficiency compared to full confinement system. Experimental observations demonstrated that the concrete at the middle distance between the FRP strips experiences more transversal expansion compared to concrete at the strip regions. It can result in a considerable decrease in the confinement performance in curtailing concrete transversal expansion, overwhelming the activation of FRP confining pressure. The present study is dedicated to the development of a new dilation model for both full and partial confinement systems, which takes into account the substantial impact of non-uniform distribution of concrete transversal expansion, a scientific topic not yet addressed comprehensibly in existing formulations. For this purpose, a reduction factor was developed in the determination of the efficiency confinement parameter, by considering available experimental results. Furthermore, based on a database of FRP fully/partially confined concrete, a new analytical relation between secant Poisson's ratio and axial strain was proposed. To evaluate the reliability and predictive performance of the developed dilation model, it was applied on the simulation of experimental tests available in the literature. The results revealed that the developed model is capable of predicting the experimental counterparts with acceptable accuracy in a design context.
\end{abstract}

Keywords: FRP confined circular concrete, Partial confinement, Dilation behavior, Axial loading,

\section{Introduction}

It is well-known that the application of fiber reinforced polymer (FRP) composite materials to externally confined concrete columns can potentially lead to substantial enhancements in terms of strength, ductility, and energy dissipation, as confirmed by studies conducted by [1-10]. Steel hoops ensure a certain concrete confinement in reinforced concrete columns. If not sufficient, concrete confinement can be enhanced by 
applying FRP strips between the existing steel hoops, resulting in a good compromise in terms of confinement efficiency and cost competitiveness for this type of structural elements [1-3]. However, the application of discrete FRP strips might pose less confinement efficiency compared to FRP fully confined concrete column (FCCC) as confirmed by [3, 7-10].

To predict the effectiveness of a FRP confining system for the improvement of concrete column responses, several theoretical models have been developed. These models are function of the relationship between axial strain and concrete transversal expansion (known as dilation behavior). Consequently, their predictive performances highly depends on the reliability of this relation. Several analytical models have been proposed to predict dilation behavior of FRP confined concrete. Mirmiran and Shahawy [5] proposed a dilation model to predict the tangential Poisson's ratio (the rate of change of transversal strain with respect to axial strain) vs axial strain relation, depending on the parameter of confinement stiffness (the ratio of confinement pressure over transversal strain). Xiao and $\mathrm{Wu}[6]$ derived a relation between secant Poisson's ratio (the ratio between transversal strain and axial strain) and axial strain as a function of unconfined concrete compressive strength and confinement stiffness. Teng et al. [11] and Lim and Ozbakkaloglu [12] proposed transversal strain vs axial strain relations dependent on the level of confinement pressure. In the case of FRP partially confined concrete column (PCCC), Zeng et al. [9] adopted Teng et al. [11]'s dilation model by applying a reduction factor in confinement pressure due to the vertical arching action. It would be noteworthy that the existing dilation models were formulated and calibrated based on FCCC specimens, therefore their applicability in the case of partial confining system is arguable. Furthermore, in the case of PCCC, the concrete at the middle distance between FRP strips, as a critical section, experiences more transversal expansion compared to concrete at the strip regions as confirmed by [7-10]. To the best of the authors' knowledge, the impact of non-uniform transversal expansion on the confinement pressure has not been addressed comprehensibly in the existing formulations. Accordingly, a generalized dilation model applicable for the both FCCC and PCCC, considering the effect of non-uniform expansion, is still lacking.

In this study, by using the results from a database of test results of FCCC and PCCC, a new dilation model with a design framework is developed that considers the FRP confinement stiffness. To account for the effect of the non-uniform distribution of the concrete expansion, a new formulation is also proposed based on the concept of confinement efficiency factor (CEF).

\section{Concept of confinement efficiency factor (CEF)}

\subsection{Original concept and its limitation when applied to $\mathrm{RC}$ columns partially confined with FRP}

During axial loading, in a PCCC system, the vertical arching action between the strips can lead to effectively and ineffectively confined concrete regions. Accordingly, the axial stress applied on the confined concrete can be assumed to be carried through two 
separate components: (1) load carried by ineffectively confined area; (2) load carried by effectively confined area. With the determination of the axial stress vs axial strain relationships of each area, the entire axial stress vs axial strain curve of PCCC can be obtained. On the other hand, for the sake of simplicity, CEF is adopted to reduce confinement stress, $f_{l}$, acting on the effectively confined area, so that the reduced confinement pressure is applied on the whole cross-section. The employed reduction CFE factor is generally represented by " $K_{e}$ ". Accordingly, the whole cross-section is uniformly subjected to an effective confinement stress $f^{\prime}{ }_{l}=K_{e} \times f_{l}$.

In the case of steel partially confined concrete, Mander et al. [13] proposed an equation to calculate $K_{e}$ as $A_{\text {eff }} / A_{g}$, where $A_{e f f}$ is the effectively confined concrete core area at the critical section (the middle distance between steel hoops) and $A_{g}$ is the whole cross-section area. Accordingly, assuming a second order parabola curve with the vertical arching angle equal to $45^{\circ}, K_{e}$ was proposed as:

$$
K_{e}=\frac{A_{e f f}}{A_{g}}=\left(1-\frac{s^{\prime}}{2 D}\right)^{2}
$$

where $D$ is the diameter of the column's circular cross section; $s^{\prime}$ is the clear distance between two adjacent steel hoops. Even though this approach has been adopted for the case of PCCC ([14-16]), a closer examination of the approach reveals that this model only addresses the effect of the vertical arching action in the determination of $K_{e}$. However, in partial systems, concrete transversal expansion at the critical section would be more than the expansion at mid-plane between consecutive steel hoops. It can result in a reduction of confinement pressure. This is not due to the negligence of Mander et al. [13], to consider the distribution of concrete expansion in the model, because confinement pressure provided by steel hoop would be constant beyond the yielding of steel. Nevertheless, in case of PCCC, due to the linear stress-strain relation of FRP material, confinement pressure developed by FRP strips directly depends on concrete expansion. Therefore, in addition to the vertical arching action, the impact of concrete transversal expansion should be addressed in the determination of $K_{e}$.

\subsection{Concrete lateral expansion}

In the present study, based on the experimental observations of PCCC [7-10], it was assumed that during axial loading, concrete at the critical section experiences the maximum transversal expansion, $\varepsilon_{l, j}$, as shown in Fig. 1. However, at the mid-plane of the FRP strips, concrete is subjected to lower dilatancy, represented by $\varepsilon_{l, i}$. If $k_{\varepsilon}$ defines the ratio of concrete expansion at the strip mid-plane and at the critical section, FRP tensile strain $\varepsilon_{h, P}$ is equal to $\varepsilon_{l, i}=k_{\varepsilon} \varepsilon_{l, j}$ (assuming that radial and hoop circumferential strains are identical). In the case of FCCC, due to a uniform distribution of concrete 
expansion, $k_{\varepsilon}$ is equal to 1 , leading to $\varepsilon_{h, F}=\varepsilon_{l, j}$. Accordingly, the ratio of FRP confining stress in PCCC and FCCC named by, $f^{\prime}{ }_{f}$ and $f_{f}$, respectively, is:

$$
\frac{f_{f}^{\prime}}{f_{f}}=\frac{E_{f} \varepsilon_{h, P}}{E_{f} \varepsilon_{h, F}}=\frac{\varepsilon_{l, i}}{\varepsilon_{l, j}}=k_{\varepsilon}
$$

As a result, the reduction factor $k_{\varepsilon}$ addresses the impact of the non-uniform distribution of concrete transversal expansion in the determination of FRP confining stress.

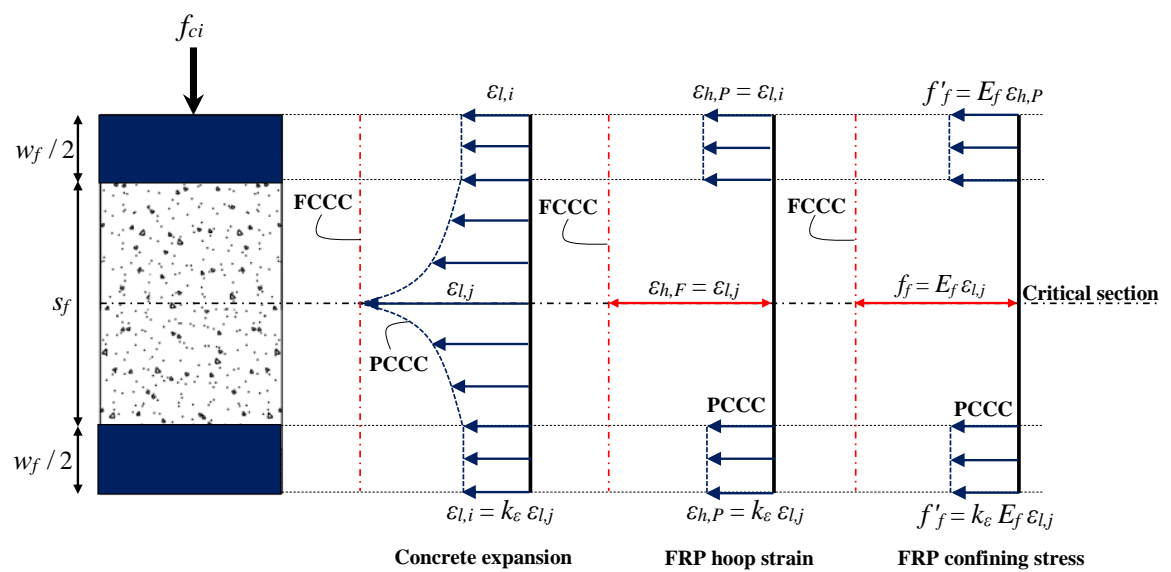

Fig. 1. Dilation behavior in PCCC system

The maximum value of this factor $\left(k_{\varepsilon, \max }\right)$ is equal to 1 for FCCC. However, for PCCC, by increasing $s_{f}, k_{\varepsilon}$ decreases until the minimum value $\left(k_{\varepsilon, \text { min }}\right)$, resulting in extensive damage around the critical section and marginal cracking at strip regions. According to the test data reported by Wang et al. [8], for $s_{f} / D \geq 1, k_{\varepsilon}$ approaches quickly to $k_{\varepsilon, \text { min }}$. Assuming a linear relation between $k_{\varepsilon}$ and $s_{f} / D$ :

$$
\begin{gathered}
k_{\varepsilon}=1-\left(1-k_{\varepsilon, \min }\right) \frac{s_{f}}{D} \geq k_{\varepsilon, \min } \\
k_{\varepsilon}=1-\left(1-k_{\varepsilon, \min }\right) \frac{s_{f}}{D} \text { for } 0 \leq s_{f} / D \leq 1
\end{gathered}
$$

To derive $k_{\varepsilon \text {,min }}$, it was assumed that concrete at the critical section is unconfined with ultimate secant Poisson's ratio $v_{s, u}$. In addition, at strip zone, concrete behaves with initial secant Poisson's ratio of unconfined concrete, $v_{s}=v_{s, 0}$. Hence, based on the dilation responses of a series of unconfined concrete specimens tested by Osorio et al. 
[17], $v_{s, u}$ and $v_{s, 0}$ were estimated approximately as 2.5 and 0.2 , respectively, therefore $k_{\varepsilon, \text { min }}$ can be calculated as:

$$
k_{\varepsilon, \min }=\frac{\varepsilon_{l, i}}{\varepsilon_{l, j}}=\frac{\varepsilon_{c} v_{s, 0}}{\varepsilon_{c} v_{s, u}}=\frac{0.2}{2.5}=0.08
$$

Fig. 2 compares the proposed relation between $k_{\varepsilon}$ and $s_{f} / D$, with the test results $[3,8-10]$. In this figure $v_{s, u}^{\prime}{ }^{\exp }$ is the ultimate secant Poisson's ratio at the mid-plane FRP strips, evaluated as the ratio of $\varepsilon_{h, P}{ }^{\text {exp }}$ recorded by strain gauge and corresponding axial strain $\varepsilon_{c}$. It should be noted that the experimental values of $k_{\varepsilon}{ }^{\text {exp }}$ are determined as $v_{s, u}^{\prime} \exp ^{\cos } 2.5$. As shown in this figure, the proposed $k_{\varepsilon}$ seems to provide relatively good agreement with the experimental test data.

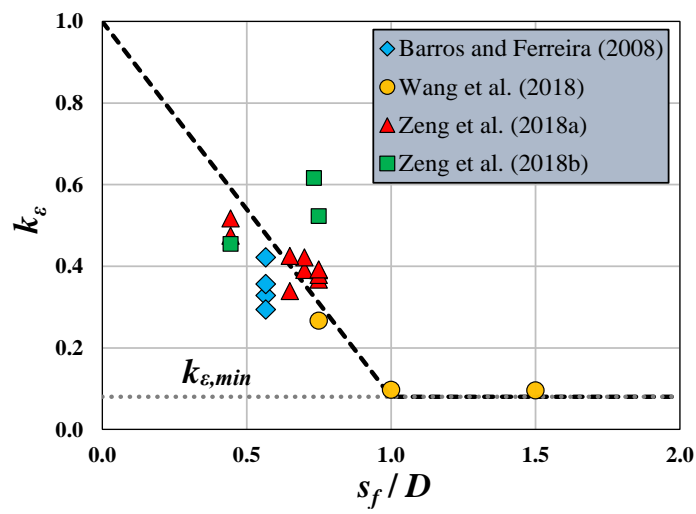

Fig. 2. Variation of $k_{\varepsilon}$ with $s_{f} / D$ obtained from Eq. (3) and the test results [3, 8-10]

\subsection{Vertical arching action}

Fig. 3 shows the non-uniform distribution of confinement pressure in a PCCC system. In this study, the reduction factor $k_{v}$ was defined so that the concrete could be considered to be evenly subjected to a reduced confinement pressure $f^{\prime}{ }_{l}=k_{v} \times f_{l, i}$. 


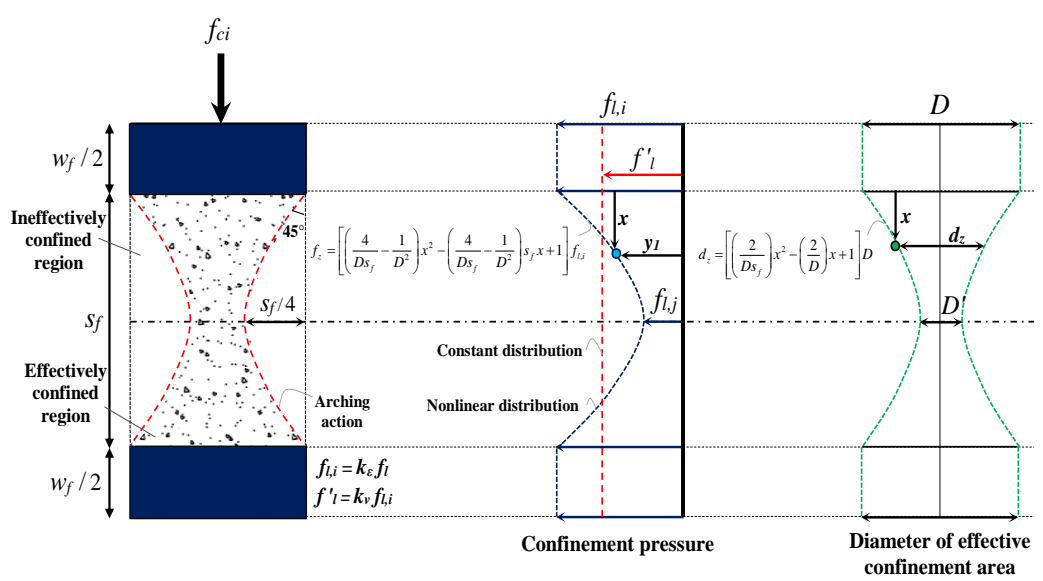

Fig. 3. Distribution of confinement pressure in PCCC

Here $f_{l, i}$ is the confinement pressure generated by FRP confining stress $f^{\prime}{ }_{f}$ at the strip region. Since confinement pressure is a function of the confining stress [13], in case of PCCC, the ratio between $f_{l, i}$ and $f_{l}$ can be expressed as:

$$
\frac{f_{l, i}}{f_{l}}=\frac{f_{f}^{\prime}}{f_{f}} \rightarrow f_{l, i}=\frac{f_{f}^{\prime}}{f_{f}} \times f_{l} \rightarrow f_{l, i}=k_{\varepsilon} \times f_{l}
$$

Therefore, the effective confinement pressure $f^{\prime}{ }_{f}$ is obtained from:

$$
f^{\prime}{ }_{l}=k_{v} f_{l, i}=k_{v} k_{\varepsilon} f_{l}=K_{e} f_{l}
$$

in which

$$
K_{e}=k_{v} k_{\varepsilon}
$$

where $K_{e}$ defines the efficiency confinement factor as a function of $k_{\varepsilon}$ and $k_{v}$, based on CEF as shown in Fig. 3. Hence, the determination of the reduction factor $k_{v}$ in Eq. (7) is necessary, as an input parameter. For this purpose, considering the equilibrium of confinement forces in a PCCC results in:

$$
k_{v} f_{l, i}\left(s_{f}+w_{f}\right) D=2 f_{l, i} \frac{w_{f}}{2} D+2 \int_{0}^{s_{f} / 2} f_{z} d_{z} d x \rightarrow k_{v}=\frac{f_{l, i} w_{f} D+2 \int_{0}^{s_{f} / 2} f_{z} d_{z} d x}{f_{l, i}\left(s_{f}+w_{f}\right) D}
$$

where $w_{f}$ is the FRP width; $f_{z}$ and $d_{z}$ are the functions of FRP lateral pressure and the diameter of effective confinement area, respectively, derived from the geometry constraint as presented in Fig. 3. Then, solving the integration yields: 


$$
k_{v}=\frac{w_{f}+s_{f}\left(1-\frac{s_{f}}{D}+\frac{13 s_{f}^{2}}{30 D^{2}}-\frac{s_{f}^{3}}{15 D^{3}}\right)}{s_{f}+w_{f}}
$$

Hence, by introducing $k_{\varepsilon}$ and $k_{v}$ obtained from Eqs. (3) and (9), $K_{e}$ can be easily calculated by Eq. (7). Based on the preliminary sensitivity analysis of the parameters in $K_{e}$, for further simplification, a simplified equation was developed as:

$$
\begin{array}{cc}
K_{e}=0.97+0.12 \frac{w_{f}}{D}-1.25 \frac{s_{f}}{D} \leq 1 & \text { for } s_{f} / D<0.5 \\
K_{e}=0.75+0.12 \frac{w_{f}}{D}-0.79 \frac{s_{f}}{D} \geq 0.04 & \text { for } 0.5 \leq s_{f} / D<1 \\
K_{e}=0.04-0.02\left(\frac{s_{f}}{D}-1\right) \geq 0 & \text { for } s_{f} / D \geq 1
\end{array}
$$

Fig. 4 shows the variation of $K_{e}$ with $s_{f} / D$ in a PCCC with $w_{f} / D=0.3$ according to Eq. (7),. It highlights the mandatory impact of $k_{\varepsilon}$ in the confinement efficiency factor $K_{e}$. Moreover, the good agreement between the results obtained from Eq. (7) and the simplified Eq. (10) confirms the reliability of the simplification. The comparison of $K_{e}$ obtained from Eq. (1) developed by Mander et al. [13] and Eq. (10), represented in Fig. 4b, also shows that the proposed model predicts lower values for $K_{e}$ than Eq. (1). It can be attributed to the consideration of the impact of $k_{\varepsilon}$, in addition to the vertical arching action, in the determination of $K_{e}$.

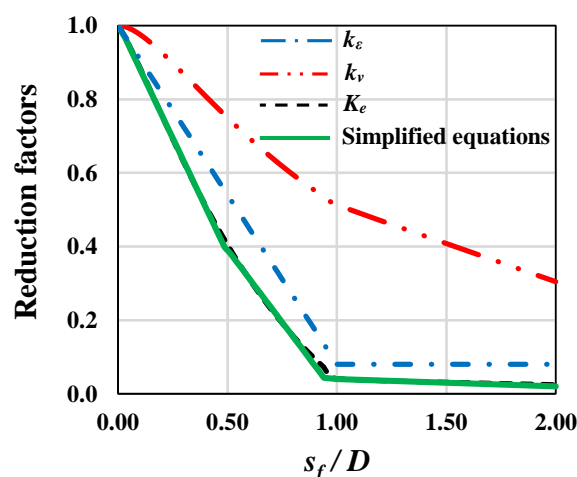

a)

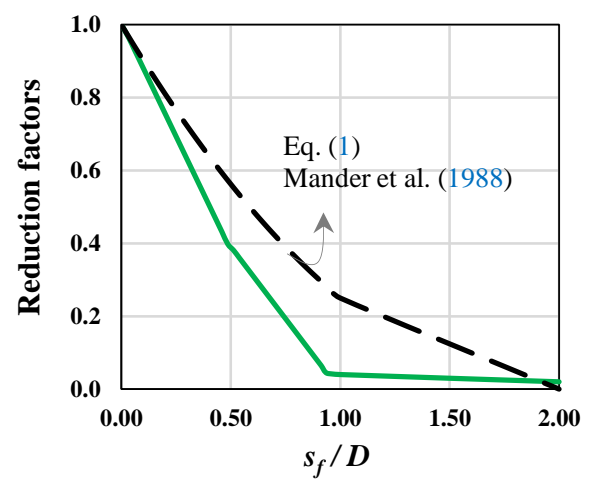

b)

Fig. 4. Variation of $K_{e}$ with $s_{f} / D$ for a PCCC system with $w_{f} / D=0.3$ 


\subsection{Effective confinement pressure}

In this section, the determination of effective confinement pressure $f^{\prime}{ }_{l}$ based on CEF will be addressed. Once confinement efficiency factor is determined, $f^{\prime}{ }_{l}$ can be calculated using equilibrium of confinement forces as $[\mathrm{X}]$ :

$$
f^{\prime}{ }_{l}=\frac{1}{2} K_{e} \rho_{f} E_{f} \varepsilon_{l, j}
$$

in which

$$
\rho_{f}=\frac{4 n_{f} t_{f} w_{f}}{\left(w_{f}+s_{f}\right) D}
$$

where $\rho_{f}$ is the FRP reinforcement ratio; $n_{f}$ and $t_{f}$ are the number and thickness of FRP layers, respectively. Considering $\varepsilon_{l, j}=v_{s} \times \varepsilon_{c}$, Eq. (11) results:

$$
f^{\prime}{ }_{l}=\frac{1}{2} K_{e} \rho_{f} E_{f} v_{s} \varepsilon_{c}
$$

Accordingly, if $\varepsilon_{c}$ is first specified, then by just addressing the corresponding $v_{s}$, effective confinement pressure $f^{\prime}$, can be calculated from Eq. (13).

\section{Determination of $v_{s}-\varepsilon_{c}$ relation}

In this section, a relation between $v_{s}$, corresponding to $\varepsilon_{l, j}$, and the applied axial compressive strain, $\varepsilon_{c}$, is determined. For this purpose, a large database consisting of 289 test specimens was set. Details of the test specimens can be found in [18]. To predict dilation behavior of both FCCC and PCCC, based on the best curve fit of the test results, the relation between $v_{s} / v_{s, \max }$ and $\varepsilon_{c}$ shown in Fig. 5 was derived, where $v_{s, \max }$ is the maximum secant Poisson's ratio at the critical section corresponding to axial strain $\varepsilon_{c, m}$ 


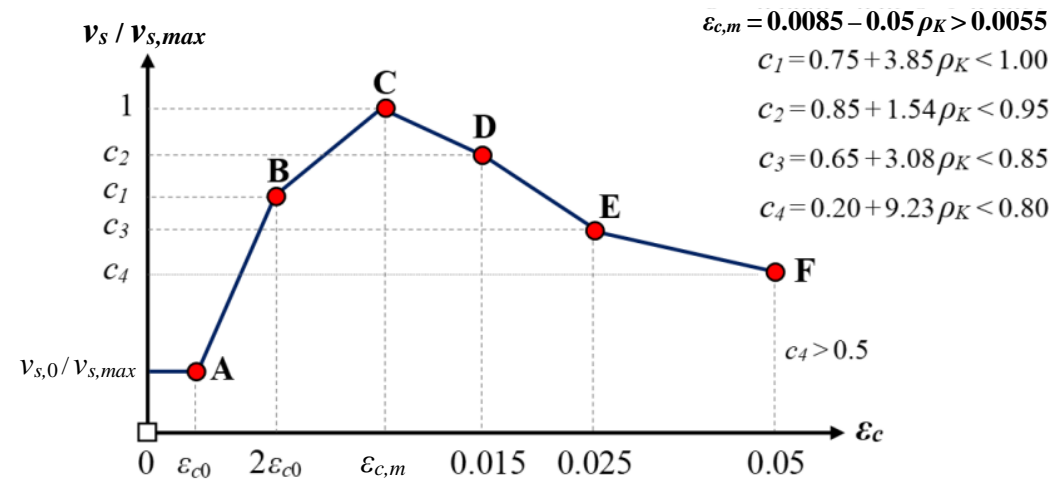

Fig. 5. Relation between $v_{s} / v_{s, \max }$ and $\varepsilon_{c}$, as a function of $\rho_{K}$

In Fig. 5, $c_{1}, c_{2}, c_{3}$ and $c_{4}$ are non-dimensional empirical coefficients derived based on the experimental results using a back analysis, and $v_{s, 0}$ is the initial Poisson's ratio of concrete, determined as suggested by Candappa et al. [19]. The parameter $\rho_{K}$, adopted to determine the previous parameters, represents the confinement stiffness index, as suggested by Teng et al. [20] for FCCC. In this study, this non-dimensional parameter index was extended for the case of PCCC by adopting the concept of confinement efficiency factor, as:

$$
\rho_{K}=\frac{f^{\prime}{ }_{l} / \varepsilon_{l, j}}{f_{c 0} / \varepsilon_{c 0}}=\frac{1}{2} K_{e} \frac{\rho_{f} E_{f}}{f_{c 0} / \varepsilon_{c 0}}
$$

As shown in Fig. 5, the expansion of confined concrete is equal to unconfined concrete up to $\varepsilon_{c}=\varepsilon_{c 0}$ (point A) with $v_{s}=v_{s, 0}$. After which, since the development of concrete cracking induces an increase in $v_{s}$, the trend enhances from $v_{s, 0}$ to $c_{1} \times v_{s, \max }$, corresponding to $\varepsilon_{c}=2 \varepsilon_{c 0}$ [13], and further up to reach $v_{s, \max }$ at $\varepsilon_{c}=\varepsilon_{c, m}$ (point C), followed by a decrease until ultimate conditions. To examine the reliability of the proposed relation, it was compared with the test results in the different levels of $\rho_{K}$, as presented in Fig. 6. There is a good agreement between the experimental and analytical results, confirming the reliability of the proposed design-based relation in Fig. 5. 

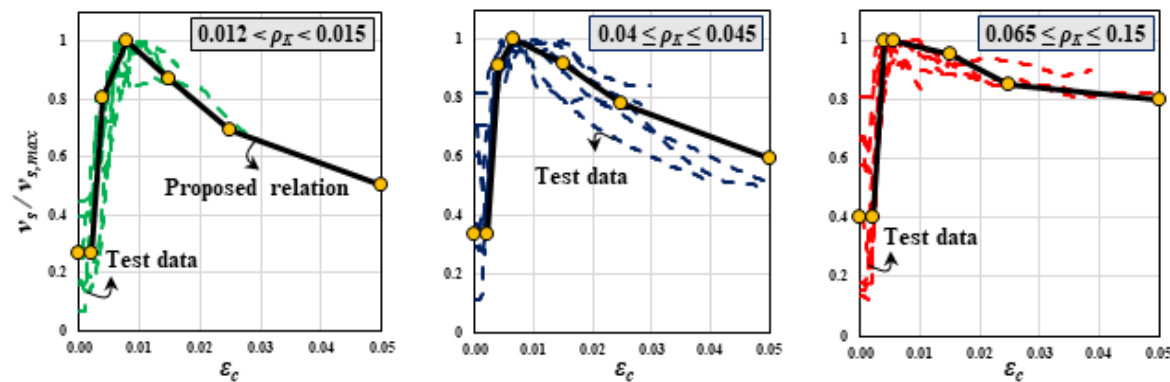

Fig. 6. $v_{s} / v_{s, \max }$ vs $\varepsilon_{c}$ determined analytically and experimentally

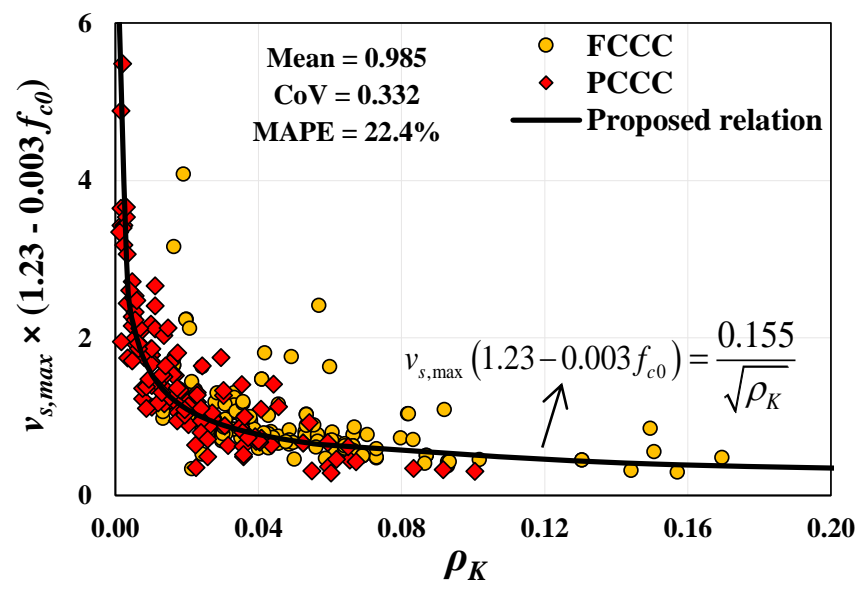

Fig. 7. Variation of the experimental $v_{s, \max }$ as a function of $\rho_{K}$

To formulate the relation between $v_{s}$ and $\varepsilon_{c}$, the determination of $v_{s, \max }$ as an input parameter is necessary. For this purpose, the following equation was determined by best curve fit of the experimental dilation results provided in the database [18] (Fig. 7):

$$
v_{s, \max }=\frac{0.155}{\left(1.23-0.003 f_{c}\right) \sqrt{\rho_{K}}}
$$

To assess its reliability for predicting $v_{s, \max }$, the results obtained from Eq. (15) are compared in Fig. 7 to those extracted from the experimental tests. The values of the mean, coefficient of variation, $\mathrm{CoV}$, and mean absolute percentage error, MAPE, reported in Fig. 7 evidences the good predictive performance of the proposed equation to estimate the value of $v_{s, \max }$ of both FCCC and PCCC. 

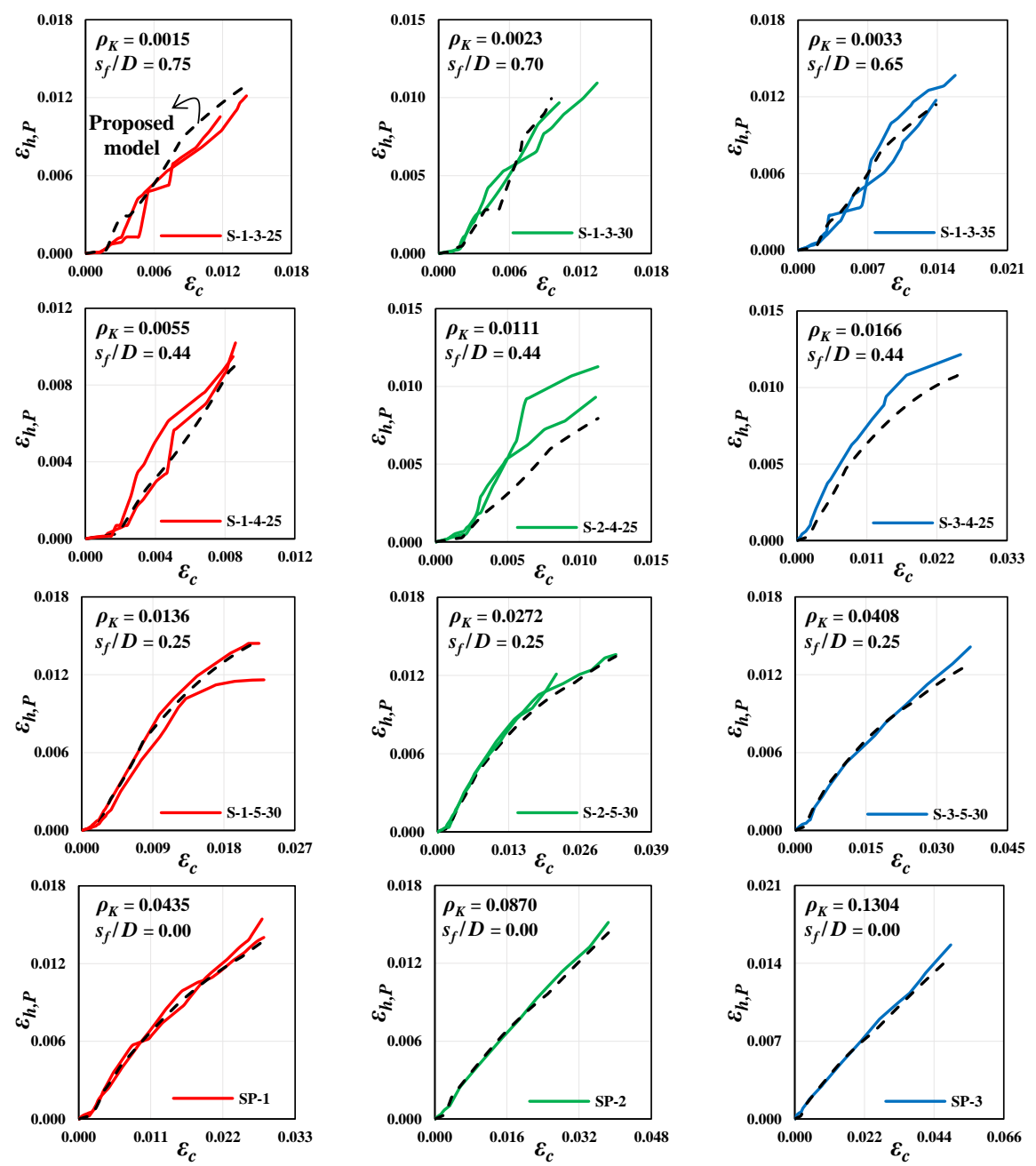

Fig. 8. Analytical vs. experimental results in terms of $\varepsilon_{h, P}$ vs $\varepsilon_{c}$ [9]

\section{$4 \quad$ Verification}

The reliability of the proposed confinement model in predicting dilation response of fully/partially FRP confined circular concrete is assessed, by considering the experimental results obtained by Zeng et al. [9] in FCCC and PCCC with different confinement configurations. Complete details of the test specimens can be found in [9]. The dilation response of the test specimens obtained experimentally and analytically from the proposed model are compared in Fig. 8, where a good predictive performance is demonstrated. 


\section{$5 \quad$ Summary and conclusions}

A new model with a design framework was developed to predict the dilation behavior of FCCC and PCCC systems. For this purpose, a relation between the secant Poisson ratio and the axial strain was proposed, dependent of the confinement stiffness. The confinement stiffness factor proposed by Teng et al. [20] was modified based on the concept of confinement efficiency factor it order to extend it to PCCC. In addition to vertical arching action, the effect of the non-uniform distribution of the concrete expansion was also considered in the determination of the efficiency confinement factor. To validate the analytical model, it was vastly applied to predict the behavior of the relevant experimental specimens available in the literature. The comparison between the model and experimental counterparts revealed that it is capable of providing an estimation of axial and dilation responses with reasonable precision in the design context.

\section{Acknowledgments}

The study reported in this paper is part of the project " StreColesf_Innovative technique using effectively composite materials for the strengthening of rectangular cross section reinforced concrete columns exposed to seismic loadings and fire', with the reference POCI-01-0145-FEDER-029485. The forth author also acknowledges the grant provided by PufProtec project with the reference POCI-01-0145-FEDER-028256.

\section{References}

1. Janwaen, W., Barros, J. A., Costa, I. G.: A new strengthening technique for increasing the load carrying capacity of rectangular reinforced concrete columns subjected to axial compressive loading. Composites Part B: Engineering 158, 67-81 (2019).

2. Perrone, M., Barros, J. A., Aprile, A.: CFRP-based strengthening technique to increase the flexural and energy dissipation capacities of RC columns. J. Compos. Constr. 13(5), 372383 (2009).

3. Barros, J. A., Ferreira, D. R. Assessing the efficiency of CFRP discrete confinement systems for concrete cylinders. J. Compos. Constr. 12(2), 134-148 (2008).

4. Shayanfar, J., Bengar, H. A.: A practical model for simulating nonlinear behaviour of FRP strengthened RC beam-column joints. Steel and Composite Structures 27(1), 49-74 (2018).

5. Mirmiran, A., Shahawy, M.: Dilation characteristics of confined concrete. Mechanics of Cohesive-frictional Materials 2(3), 237-249 (1997).

6. Xiao, Y., Wu, H.: Compressive behavior of concrete confined by various types of FRP composite jackets. Journal of Reinforced Plastics and Composites 22(13), 1187-1201 (2003).

7. Guo, Y. C., Gao, W. Y., Zeng, J. J., Duan, Z. J., Ni, X. Y., Peng, K. D.: Compressive behavior of FRP ring-confined concrete in circular columns: Effects of specimen size and a new design-oriented stress-strain model. Construction and Building Materials 201, 350-368 (2019).

8. Wang, W., Sheikh, M. N., Al-Baali, A. Q., Hadi, M. N.: Compressive behaviour of partially FRP confined concrete: Experimental observations and assessment of the stress-strain models. Construction and Building Materials 192, 785-797 (2018). 
9. Zeng, J. J., Guo, Y. C., Gao, W. Y., Chen, W. P., Li, L. J.: Stress-strain behavior of concrete in circular concrete columns partially wrapped with FRP strips. Composite Structures 200, 810-828 (2018).

10. Zeng, J., Guo, Y., Li, L., Chen, W.: Behavior and three-dimensional finite element modeling of circular concrete columns partially wrapped with FRP strips. Polymers 10(3), 253 (2018).

11. Teng, J., Huang, Y. L., Lam, L., Ye, L. P.: Theoretical model for fiber-reinforced polymerconfined concrete. J. Compos. Constr. 11(2), 201-210 (2007).

12. Lim, J. C., Ozbakkaloglu, T.: Lateral strain-to-axial strain relationship of confined concrete. Journal of Structural Engineering 141(5), 04014141 (2014).

13. Mander, J. B., Priestley, M. J., Park, R.: Theoretical stress-strain model for confined concrete. Journal of structural engineering 114(8), 1804-1826 (1988).

14. Fib Bulletin 14.: Externally bonded FRP reinforcement for RC structures. International Federation for Structural Concrete (2001).

15. ACI-318-08.: Building code requirements for structural concrete, in American Concrete Institute. 2008, American Concrete Institute: Farmington Hills MI (2008).

16. CNR-DT 200.: Guide for the design and construction of externally bonded FRP systems for strengthening existing structures. Italian National Research Council (2004).

17. Osorio, E., Bairán, J. M., Marí, A. R.: Lateral behavior of concrete under uniaxial compressive cyclic loading. Materials and Structures 46(5), 709-724 (2013).

18. Shayanfar, J., Rezazadeh, Barros J. A., Ramezansefat H.: A new theoretical model to predict axial and dilation responses of FRP fully/partially confined circular concrete. Research Report, ISISE, Department of Civil Engineering, University of Minho, Portugal, (2020).

19. Candappa, D. C., Sanjayan, J. G., Setunge, S.: Complete triaxial stress-strain curves of highstrength concrete. Journal of Materials in Civil Engineering 13(3), 209-215 (2001).

20. Teng, J. G., Jiang, T., Lam, L., Luo, Y. Z.: Refinement of a design-oriented stress-strain model for FRP-confined concrete. J. Compos. Constr. 13(4), 269-278 (2010). 